\section{Rhinitis: von der Lokal- zur Systemallergie}

\begin{abstract}
Bei der sogenannten nicht allergischen Rhinitis (NAR) sind Pricktest und Nachweis von spezifischem IgE negativ. Häufig können aber in der Nasenschleimhaut lokal IgE-Antikörper nachgewiesen werden. Ist das der Ausgangspunkt für den Weg zur systemischen Allergie?
\end{abstract}

$D^{i}$ ie NAR gehört zur Gruppe der idiopathischen Rhinitiden, bei denen das symptomauslösende Agens unbekannt bleibt. Die meisten Betroffenen haben persistierende Symptome und häufig ein begleitendes Asthma. Bei Patienten mit NAR wurden aber auch IgEAntikörper in der Nasenschleimhaut gefunden, es wird hier von einer "lokalen“ Allergie oder „Entopie“ gesprochen.

In einer Studie an einem spanischen Allergiezentrum wurden 180 Patienten mit der Diagnose einer NAR in den Jahren 2000 bis 2004 mehrere Jahre später (2007) nochmals untersucht. 52\% der Patienten hatten eine Verschlechterung ihrer Erkrankung festgestellt, bei 12\% war die Beschwerdedauer verlängert, bei 9\% hatten sich die nasalen Symptome verschlimmert und bei $24 \%$ waren neue
Erkrankungen dazu gekommen. Die häufigsten Komorbiditäten waren Asthma und Konjunktivitis, wobei die

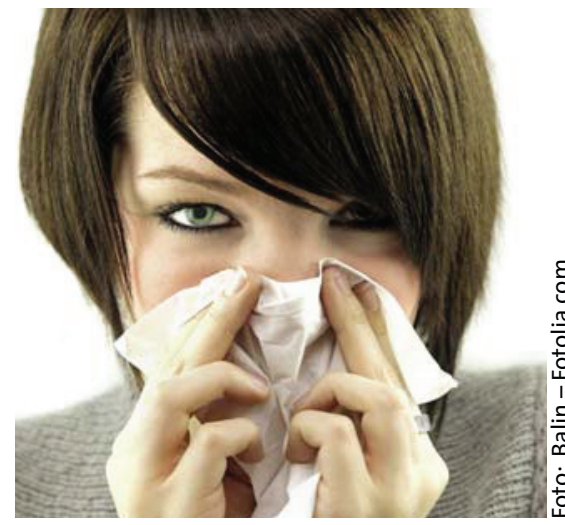

Bei zunächst nicht allergischer Rhinitis sind wiederholte Allergietests zu empfehlen.
Asthmainzidenz von $32 \%$ auf $55 \%$ und die Konjunktivitisinzidenz von $28 \%$ auf $43 \%$ gestiegen waren. Bei $8 \%$ der NARPatienten hatten sich die Symptome gebessert, in keinem Fall waren die Rhinitissymptome jedoch verschwunden. Bei der Erstvorstellung der Patienten waren die allergologischen Tests negativ ausgefallen. Nun zeigten $24 \%$ der Patienten eine Sensibilisierung gegen Aeroallergene, vor allem gegen Pollen und Hausstaubmilbe - nachgewiesen im Hauttest oder durch spezifisches Serum-IgE.

Fazit: Bei Patienten mit der Diagnose einer NAR verschlechtert sich die Erkrankung im Verlauf einiger Jahre deutlich. Bei einem Teil der Patienten können im Verlauf Sensibilisierungen nachgewiesen werden. Patienten mit NAR sollten deshalb in regelmäßigen Abständen einer erneuten allergologischen Diagnostik unterzogen werden. $\quad b k$

Rondón C et al. Evolution of patients with nonallergic rhinitis supports conversion to allergic rhinitis. J Allergy Clin Immunol 2009; 123: 1098-102

\title{
Bakterielle Infektion und Allergie
}

Nach der Hygienehypothese hat der frühe Kontakt mit mikrobiellen Antigenen einen protektiven Effekt bzgl. der Entwicklung allergischer Erkrankungen. In einer aktuellen Studie wurde nun der Einfluss einer Chlamydieninfektion auf die allergische Reaktion untersucht.

$\mathrm{n}$ einer tierexperimentellen Studie wurden Mäuse im Alter von acht bis zwölf Wochen mit Chlamydia pneumoniae in zwei unterschiedlichen Dosierungen $\left(0,5\right.$ bzw. $5 \times 10^{6}$,inclusion forming units", IFU) infiziert. Danach erfolgte eine erste intranasale Applikation von humanem Serumalbumin an drei aufeinander folgenden Tagen (Sensibilisierung). Knapp zwei Wochen später bekamen die Tiere erneut Serumalbumin verabreicht (Reexposition), nach einer weiteren Woche wurden Lungengewebe sowie das Serum der Tiere untersucht.

Tiere, die eine niedrige Bakteriendosis erhalten hatten, zeigten eine Entzündungsreaktion der Lunge mit Eosinophilie und Becherzellhyperplasie.
Die Höhe von Gesamt-IgE, spezifischem $\mathrm{IgE}$ und $\mathrm{IgG}_{1}$ im Serum waren mit dem Grad der Eosinophilie assoziiert. Bei der höheren Bakteriendosis dagegen konnte keine Zunahme von Eosinophilen und Becherzellen nachgewiesen werden. Spezifisches IgE und $\mathrm{IgG}_{1}$ stiegen hier auch weniger deutlich an. Zusätzlich wiesen nur diejenigen Mäuse Eosinophilie, Becherzellhyperplasie und Albumin-spezifische Antikörper auf, die bereits fünf Tage nach der (niedrig dosierten) Chlamydieninfektion mit dem Allergen sensibilisiert worden waren. Erfolgte die erste Exposition mit Albumin erst zehn Tage nach der Infektion, war diese Reaktion nicht nachweisbar.
In einem interessanten Ansatz wurden nun dendritische Zellen von infizierten Mäusen isoliert, mit Albumin inkubiert und danach Albumin-naiven Mäusen intratracheal übertragen. Die Empfänger entwickelten 15 Tage später beim ersten Kontakt mit Albumin eine Entzündungsreaktion der Lunge mit Eosinophilie und Becherzellhyperplasie. Mäuse, die dendritische Zellen von nicht infizierten Tieren erhielten, zeigten keine Entzündungsreaktion.

Fazit: In dieser Studie zeigte sich, dass die Infektion mit Chlamydia trachomatis eine eosinophile Reaktion nach einer Sensibilisierung induzieren kann. Dabei sind die Anzahl der Erreger und das Zeitintervall zwischen Infektion und Allergenexposition entscheidend. af

Schröder NW et al. Innate immune responses during respiratory tract infection with a bacterial pathogen induce allergic airway sensitization. J Allergy Clin Immunol 2008; 122: 595-602 